\title{
Predicting Survival in Patients with Glioblastoma Using MRI Radiomic Features Extracted from Radiation Planning Volumes
}

\section{Benjamin Geraghty}

Sunnybrook Health Sciences Centre

Archya Dasgupta

Sunnybrook Health Sciences Centre

Michael Sandhu

Sunnybrook Health Sciences Centre

Nauman Malik

Sunnybrook Health Sciences Centre

Pejman Jabehdar Maralani

Sunnybrook Health Sciences Centre

Jay Detsky

Sunnybrook Health Sciences Centre

Chia-Lin Tseng

Sunnybrook Health Sciences Centre

Hany Soliman

Sunnybrook Health Sciences Centre

Sten Myrehaug

Sunnybrook Health Sciences Centre

Zain Husain

Sunnybrook Health Sciences Centre

James Perry

Sunnybrook Health Sciences Centre

Angus Lau

Sunnybrook Health Sciences Centre

Arjun Sahgal

Sunnybrook Health Sciences Centre

Gregory Czarnota ( $\nabla$ gregoryczarnota.submissions@gmail.com )

Sunnybrook Health Sciences Centre https://orcid.org/0000-0002-0519-2182 
Keywords: Radiomics, Glioblastoma multiforme (GBM), Magnetic Resonance Imaging (MRI), Radiotherapy.

Posted Date: November 17th, 2021

DOI: https://doi.org/10.21203/rs.3.rs-1078827/v1

License: (c) (1) This work is licensed under a Creative Commons Attribution 4.0 International License. Read Full License

Version of Record: A version of this preprint was published at Journal of Neuro-Oncology on January 3rd, 2022. See the published version at https://doi.org/10.1007/s11060-021-03939-9. 


\section{Abstract}

Background: Quantitative image analysis using pre-operative magnetic resonance imaging (MRI) has been able to predict survival in patients with glioblastoma (GBM). The study explored the role of postoperative radiation (RT) planning MRI-based radiomics to predict the outcomes, with features extracted from the gross tumor volume (GTV) and clinical target volume (CTV).

Methods: Patients with IDH-wildtype GBM treated with adjuvant RT having MRI as a part of RT planning process were included in the study. 546 features were extracted from each GTV and CTV. A LASSO Cox model was applied, and internal validation was performed using leave-one-out cross-validation with overall survival as endpoint. Cross-validated time-dependent area under curve (AUC) was constructed to test the efficacy of the radiomics model, and clinical features were used to generate a combined model. Analysis was done for the entire group and in individual surgical groups-gross total excision (GTR), subtotal resection (STR), and biopsy.

Results: 235 patients were included in the study with 57, 118, and 60 in the GTR, STR, and biopsy subgroup, respectively. Using the radiomics model, binary risk groups were feasible in the entire cohort $(p<0.01)$ and biopsy group $(p=0.04)$, but not in the other 2 surgical groups individually. The integrated AUC (iAUC) was 0.613 for radiomics-based classification in the biopsy subgroup, which improved to 0.632 with the inclusion of clinical features.

Conclusion: Imaging features extracted from the GTV and CTV regions can lead to risk-stratification of GBM undergoing biopsy, while it was redundant for patients with GTR and STR.

\section{Introduction}

Radiomics involve quantitative image analysis aided by computer vision with an emerging role in medicine and oncology as noninvasive biomarkers [1]. The high-dimensional imaging data can be linked to a diverse range of biological and clinical endpoints, including histological diagnosis, molecular characteristics, treatment response, survival outcomes, and toxicity estimation [2]. Magnetic resonance imaging (MRI) is considered the gold standard imaging modality in central nervous system (CNS) tumors due to superior anatomical representation of intracranial structures compared to other imaging modalities as well functional information from advanced sequences like perfusion, diffusion, spectroscopy [3]. Radiomics research has been widely applied in CNS tumors including gliomas in characterizing tumor histology (astrocytoma vs. oligodendroglioma), tumor grade (low grade vs. high grade), risk-stratification, and quantitative response assessment $[4,5]$.

Glioblastoma (GBM) is a grade 4 glial neoplasm, associated with poor prognosis with a median survival of 15 months [6]. Following maximal safe resection, patients are treated with radiotherapy (RT) along with concurrent temozolomide. Radiation target volumes constitute gross tumor volume (GTV) and the clinical target volume (CTV) accounting for macroscopic residual disease along with surgical cavity and microscopic infiltrative disease in the peritumoral region, respectively [7]. Following RT completion, 
patients are treated with adjuvant temozolomide and serial interval MRIs are performed to monitor disease status. As a part of RT planning, patients undergo computed tomography (CT) for dose computation purposes and MRI for delineation of target volumes and organs at risk. A significant body of work has been undertaken utilizing pre-operative MRI for patients with GBM, demonstrating the feasibility of prognostication and identifying different subsets of patients with variable outcomes [4]. However, limited data is available using radiomics on post-surgery or pre-radiation imaging, which can tentatively provide important insights into biological behavior. In this study, we have undertaken exploratory analysis to investigate the impact of quantitative features obtained from RT planning MRI, as well as the impact across different surgical resection subsets.

\section{Methods}

\section{Patient Selection}

Patients with newly diagnosed IDH-wildtype GBM treated with RT at Sunnybrook Health Sciences Centre, Toronto, between January 2014 and December 2018 were considered eligible for the retrospective study. The institutional ethics committee approved the study, and the requirement for informed consent were waived. Patients who had T1-weighted gadolinium contrast-enhanced (T1-CE), T2-weighted FLAIR (T2FLAIR), and apparent diffusion coefficient (ADC) MRI sequences and completed the scheduled course of RT were considered eligible for the study. Patients with prior RT, missing survival information, missing or motion artifact corrupted images were excluded.

\section{Radiation Planning Contour Extraction and Image Preprocessing}

Patients were treated with RT and chemotherapy following surgery according to standard protocols $[6,8]$. Following consultation and decision for RT, planning CT and MRI was performed for all patients. Radiation planning MRIs were acquired on GE Signa HDxT 1.5T MRI scanners (GE Medical Systems, WI, USA) or Philips Ingenia 1.5T systems (Philips Medical, WI, USA). The slice thickness for T1-CE, T2-FLAIR, and ADC was $1 \mathrm{~mm}, 2 \mathrm{~mm}$, and $5 \mathrm{~mm}$, respectively. The RT target volumes were drawn by the responsible radiation oncologists. GTV encompassed the enhancing residual disease on the T1-CE sequence and the surgical cavity. CTV included a $1.5 \mathrm{~cm}$ expansion beyond the GTV typically encompassing the PTR identified as hyperintense region on T2-FLAIR, duly edited from anatomical barriers like the falx, tentorium cerebelli, and bone. A planning target volume of $5 \mathrm{~mm}$ was used. All patients were treated on a linear accelerator device using intensity-modulated RT with image guidance. Typical dose prescriptions included $60 \mathrm{~Gy}$ in 30 fractions over 6 weeks in patients less than 65 years or $40 \mathrm{~Gy}$ in 15 fractions over 3 weeks in elderly patients or patients with poor performance status. The decision regarding concurrent and adjuvant temozolomide was taken by the responsible neuro-oncologist and radiation oncologist.

Contours for the GTV and CTV that were planned on fused CT/T1-CE images in Pinnacle (Philips Medical, WI, USA) were exported into Matlab R2018b (The Mathworks, Inc., MA, USA), where the CT-based contours were transformed to the T1-CE reference frame using the CT/T1-CE registration information. Images were skull stripped using HD-BET [9], and then the T2-FLAIR and ADC volumes were rigidly registered to the 
corresponding T1-CE volumes using the FMIRB Software Library (FSL) tool FLIRT [10-12]. The contours and images were resampled to a uniform resolution of $1 \mathrm{~mm}^{3}, \mathrm{~N} 4$ bias field corrected $[13,14]$ and intensity normalized by histogram matching [15-17] to the T1-CE and T2-FLAIR images acquired on the GE scanners. Finally, the CTV contours were manually refined using ITK-SNAP [18] (http://www.itksnap.org) to omit the ventricles, prominent sulci, and the skull where applicable, and the GTV was subtracted from the CTV to generate two disjoint contours.

\section{Feature Extraction}

Radiomic features were extracted from the GTV and modified CTV for each of the three MRI sequences. Fixed bin width (FBW) quantization was used to discretize pixel intensities within each contour. To determine the FBW for each modality and contour type, the minimum and maximum intensities were measured, and the FBW was selected as the maximum width that produced bin counts greater than or equal to 30. Feature extraction was performed using PyRadiomics [19] software V2.2.0. The feature set included the following: 18 first-order statistical features; 22 gray level co-occurrence matrix features; 16 gray level size zone features; 16 gray level run length matrix features; 5 neighboring gray tone difference matrix features; and 14 gray level dependence matrix features. Ninety-one features were extracted for the GTV and CTV for each of the three MR modalities, resulting in a total of 546 radiomic features per patient. A detailed description of the features can be found on the PyRadiomics website (https://pyradiomics.readthedocs.io). A schematic of the data processing is shown in Figure 1.

\section{Statistical Analysis}

\section{Risk Modelling}

The statistical analysis was performed using R V4.0.3 [20] (http://www.R-project.org). Four independent models were considered: one including all patients and one for each of the three surgical treatment subgroups i.e., gross total resection, subtotal resection, and biopsy. Internal validation was performed using leave-one-out cross-validation. First, a patient was left out, and the radiomic features from the remaining patients were shifted and scaled to zero mean and unit standard deviation using the caret [21] package function "PreProcess", and then the shifting/scaling parameters were applied to the test patient. Using the package $g / m n e t[22,23]$, the radiomics signatures were constructed by least absolute shrinkage and selection operator (LASSO) Cox regression. To reduce potential overfitting, the regularization weight $\lambda$ was optimized to minimize the 10 -fold cross-validation error on the training set. The fitted LASSO Cox model was then applied to the training and test patients and radiomics risk scores were derived by a linear combination of the features weighed by their model coefficients. The median of the training patients' radiomics risk scores was used as a threshold to assign the test patient to either low or high risk. Using the survival $[24,25]$ package, Cox proportional hazard models were fit to the clinical features of the training data, which included age, GTV volume, and surgical treatment, and linear predictions were made on the test patient to produce a clinical risk score. Additionally, a combined radiomics and clinical Cox model was fit on the training data and applied to the test patient to create a combined risk score. All 
modeling steps were repeated from scratch for each left-out patient. A flowchart of the leave-one-out cross-validation procedure is shown in Supplementary Figure 1.

\section{Evaluation of Predictive Accuracy}

Cross-validated Kaplan-Meier curves for high/low overall survival (OS) risk were constructed using the survival package function "survfit." The split into binary risk groups (high and low) were obtained using the major split as obtained from the algorithm. The date of surgery was considered as baseline for the survival analysis. To assign statistical significance to the log-rank tests, the permutation distributions of the log-rank statistics were obtained by randomly permuting the correspondence between radiomic features and OS and repeating the entire leave-one-out cross-validation procedure 100 times. To test the hypothesis of predicting OS using radiomic features, the proportion of permuted log-rank statistics greater than or equal to the un-permuted statistic was taken as the significance level [26]. A weighted logrank test was used ( $G$-rho rank test, rho $=1$ ). The cross-validated time-dependent area under the receiver operator characteristic curves for the radiomics, clinical, and combined risk scores were generated using the package risksetROC [27], and the integrated areas under the time-dependent curves (iAUC) were evaluated with the function "risksetAUC." To evaluate the incremental value of radiomics to clinical in OS prediction, the null hypothesis that radiomics are independent of OS and clinical variables was tested by obtaining the permutation distribution of the iAUCs. In this case, the correspondence between radiomic features and OS were randomly permuted while clinical variables were left un-permuted and the leaveone-out cross validation procedure was repeated 100 times. The difference in iAUC measures between the combined and clinical models was used as the test metric. The proportion of iAUC differences with permuted radiomic features greater than or equal to the un-permuted iAUC differences were taken as the significance level. Kendall's $\tau_{b}$ was used to test the association between the predicted radiomic risk scores and surgical treatment. Normality of the radiomics risk scores was assessed with the Shapiro-Wilk test and a one-way ANOVA or Kurskal-Wallis test for risk scores by surgical treatment was used where appropriate. $\mathrm{p}$-values $<0.05$ were taken as significant.

\section{Results}

\section{Clinical Characteristics}

A total of 235 patients were included in the study. Patients undergoing gross total resection (GTR), subtotal resection (STR), and biopsy were $24 \%, 50 \%$, and $26 \%$ of the cohort, respectively. The median interval between surgery and planning MRI was 19 days. The six week long RT course was delivered to $56 \%$ of patients. Concurrent and adjuvant temozolomide was used in $91 \%$ and $65 \%$ of patients, respectively. The median OS for all patients was 13 months. The detailed clinical and treatment characteristics for all patients as well as the surgical treatment subgroups are listed in Table 1.

\section{Radiomics Risk Stratification}


The cross-validated Kaplan-Meier survival curves for classifying patients into low- or high-risk groups according to the radiomics signatures for each of the four patient sub-groupings are shown in Figure 2. The radiomics signatures derived from the inclusion of all surgery subtypes stratified the patients into low-risk and high-risk groups with G-rho permutation test p-value $<0.01$. For the GTR and STR models, the radiomics signature could not stratify risk groups with statistical significance. In the biopsy subgroup model, the radiomics signature stratified patients into low-risk and high-risk groups $(p=0.04)$. A boxplot of the predicted radiomics risk scores as well stratified by surgical treatment are shown in Figure 3 , showing significantly higher risk in patients with a higher residual disease post-surgery (biopsy>STR>GTR).

\section{Survival Prediction}

The cross-validated time-dependent area under the receiver operator characteristic curves of the radiomic, clinical, and combined models for survival prediction in the patient subgroups are shown in Figure 4. For the prediction models including all patients, the iAUCs of the radiomics signature was 0.593 ; the clinical model was 0.644; and the combined radiomics and clinical model was 0.632. The iAUC difference ( $\mathrm{iAUC}_{\text {combined }}-\mathrm{iAUC} \mathrm{Clinical}$ ) was -0.012 . For the GTR subgroup, the iAUCs of the radiomics signature were 0.576; the clinical model was 0.614; and the combined model was 0.604 , with an iAUC difference of -0.01 . For the STR subgroup, the iAUC of the radiomics signature was 0.540 ; the clinical model was 0.538 ; and the combined model was 0.523 . The iAUC difference was -0.015 . Finally, for the biopsy subgroup, the iAUC of the radiomics signature was 0.613 ; the clinical model was 0.522 ; and the combined model was 0.632. The iAUC difference was 0.11 (permutation test $p<0.01$ ).

Analysis of the radiomics risk scores derived from the inclusion of all patients showed a moderate to strong association of radiomics risk with surgical treatment $\left(\tau_{b}=-0.343, P \ll 0.01\right)$. The Kruskal-Wallis test revealed a significant difference in the predicted radiomics risks among surgical treatment groups ( $p$ $<0.001)$ and a large effect size $\left(\eta^{2}=0.188\right)$. Post-hoc multiple pairwise comparisons using Wilcoxon's test with Bonferroni $p$-value adjustment revealed significant differences between the biopsy and STR groups $(P<0.01)$; between the biopsy and GTR groups $(p<<0.01)$; as well as between the STR and GTR groups ( $p<<0.01$ ). A G-rho rank test of OS for the surgical treatment groups showed a significant difference in OS ( $p<0.01$ ). The survival plot for OS curves stratified by surgical treatment is shown in

\section{Supplementary Figure 2.}

\section{Discussion}

Computational analysis techniques have enabled medical imaging to cross the boundaries of traditional contribution in diagnostics and serve as noninvasive biomarkers [28]. Since MRI has an integral role at different stages of management of CNS tumors, quantitative analysis provides an excellent opportunity for integration with clinical factors to improve prognostication as well as potential treatment modifications. In this study, we have undertaken radiomic analysis of three MRI sequences extracted 
from the standard radiation target volumes performed following surgery as a part of RT planning to predict overall survival.

Radiation oncology treatment planning and delivery are deeply intertwined with various morphological and functional imaging modalities needed for dosimetry computation, delineation of target volumes and avoidance structures, and guiding treatment delivery. Although cone-beam CT images during RT have been analyzed in different malignancies like lung or head-neck primaries $(26,27)$, there is a dearth of studies regarding the use of pre-treatment RT volumes and corresponding imaging data in radiomic analysis. As the general principle for RT is the presence of macroscopic tumors within GTV and microscopic disease within the CTV, a significant amount of biological information is expected to be contained within the target volumes. It is reasonable to hypothesize that the GTV is likely to contain more information than CTV volumes when RT is used as the primary modality rather than an adjuvant treatment. Also, in such a scenario, the imaging parameters obtained following surgery might turn out challenging with postoperative changes (e.g. gliosis, blood products in the brain), causing obfuscation of underlying biological information. In patients with GBM, although radiation is considered in the adjuvant setting, the surgical principle includes maximal safe resection, with macroscopic residual disease often left behind in proximity to eloquent areas. Therefore, we had considered performing analysis according to the surgical subgroups to understand the impact of residual disease. Also, it is well known the presence of microscopic infiltrative disease in the peritumoral region (PTR) surrounding the enhancing disease in patients with GBM, which is included within the CTV. Previous work has shown the clinical utility of radiomic analysis of PTR in GBM, with Prasanna et al. demonstrating quantitative features extracted from the PTR region alone using pre-operative MRI was able to stratify patients with different survival outcomes [31]. In our previous work, we have demonstrated voxel-based mapping to identify areas with infiltrative disease in the PTR, which correlated with areas of future recurrence [32]. Also, the distinctive MRI characteristics of the GBM PTR (constituting microscopic disease and edema) as opposed to low grade glioma (composed of infiltrative tumor cells) have been demonstrated using radiomics approach [33].

In the present study, the radiomic features extracted from the radiation target volumes were able to stratify the survival outcomes into two groups for all the 235 patients $(p<0.01)$ and biopsy subgroups (0.04). In the other two surgical subgroups (GTR and STR), risk-stratification was not possible, which can be justified by the absence of enough biological information contained with the target volumes (due to the removal of major bulk of macroscopic disease). When the clinical features were included in the radiomics model, the combined model performed significantly better (iAUC $=0.632$ ) as compared to the radiomics model ( $\mathrm{A} A U \mathrm{C}=0.613$ ) or clinical model ( $\mathrm{i} A U \mathrm{C}=0.522)$ alone. The findings from our study are quite similar to the radiomics study of pre-operative MRI, where the iAUC for radiomics model alone for overall survival and progression-free survival was 0.652 and 0.590 , respectively, which improved to some extent with the use of clinical features [34]. The radiomics risk score was significantly better in the biopsy group than the other two subgroups indicating the presence of macroscopic tumor or core (corresponding to GTV) was the driver of the classifiers rather than the microscopic disease or the PTR (corresponding to CTV) in the postoperative setting. One important consideration here is the CTV in the majority of 
instances was not only limited to the T2 hyperintensity but also included the normal signal contained within the isotropic expansion from GTV edges, which could potentially weaken the relevant imaging information.

It may be worthwhile to pursue future studies to limit the feature extraction to only areas of altered FLAIR signal intensity within the CTV volume. A recently published phase $1 / 2$ trial by Azoulay et al. used a small CTV margin of $5 \mathrm{~mm}$ with hypofractionated RT resulting in equivalent clinical outcomes in GBM, challenging the traditional concept of larger margins and possibly reducing toxicity [35]. The generation of efficient imaging biomarkers from RT planning MRI can pave the way towards precision radiation oncology workflow tailoring treatment protocols individually, such as treatment-escalation in high-risk groups. The advent of MR-LINAC in clinical practice has generated an opportunity to generate and adopt imaging biomarkers not only before treatment initiation but also to study the temporal changes during the course of RT. The prospective UNITED trial investigating the role of MR-LINAC guided small-margin adaptive RT (clinicaltrials.gov identifier NCT04726397) provides an opportunity to evaluate changes in radiomic features throughout a course of $\mathrm{RT}$, which may provide additional insight in terms of survival prediction. Another limitation was the exclusion of MGMT from the clinical model, due to unavailability in a proportion of patients, which is otherwise known to strongly influence the survival outcomes.

\section{Conclusion}

Radiomic features from radiation planning T1 CE, T2-FLAIR, and ADC MRI sequences corresponding to the GTV and CTV can be used for predicting survival in patients with GBM, particularly in the subgroup undergoing biopsy. The iAUC of 0.613 was achieved for a radiomics-based classification for predicting OS in the biopsy subgroup, which improved to 0.632 with the inclusion of clinical features.

\section{Declarations}

Acknowledgments: We express our sincere gratitude to the patients and their caregivers involved in the study. We would like to thank the Terry Fox Foundation Program Project Grant from the Hecht Foundation for the funding support associated with the study

Funding: Terry Fox Foundation Program Project Grant from the Hecht Foundation (1083) awarded to Gregory J. Czarnota. The funding bodies had no influence on the study design, data collection, analysis, interpretation of data, or the manuscript's writing.

\section{Conflict of interest:}

Benjamin Geraghty: None

Archya Dasgupta: None

Michael Sandhu: None 
Nauman Malik: None

Pejman Jabehdar Maralani: None

Jay Detsky: None

Chia-Lin Tseng: None

Hany Soliman: None

Sten Myrehaug: None

Zain Husain: None

James Perry: None

Angus Lau: None

Arjun Sahgal:

Research grant with Elekta $A B$, Varian

Past educational seminars with Elekta (Gamma Knife Icon) and Elekta AB, Accuray Inc., Varian (Medical Advisory group and CNS Teaching Faculty), BrainLAB, AstraZeneca, Medtronic Kyphon

Travel accommodations/expenses by Elekta, Varian, BrainLAB

Board Member: International Stereotactic Radiosurgery Society (ISRS)

Co-chair with AO Spine Knowledge Forum Tumor

Elekta MR Linac Research Consortium, Elekta Spine, Oligometastases and Linac Based SRS Consortia

Gregory J. Czarnota: Funding received from the Terry Fox Foundation Program Project Grant.

Availability of data and material: Data are stored in an institutional repository and will be made available on request to the corresponding author following institutional ethics committee protocols.

Code availability: The radiomic feature extraction was performed using freely available Pyradiomics software (http://www.pyradiomics.io/pyradiomics.html).

\section{Author contributions:}

Conceptualization: GJC, BG, AD, AS; Methodology: All authors; Formal Analysis and investigation: All authors; Writing-original draft preparation: $B G, A D, A S, G J C$; Writing-review and editing: All authors; Project administration and supervision: AS, GJC; Funding acquisition: GJC 
All the authors are in agreement and accountable for all the aspects of the work.

\section{References}

1. Lambin P, Rios-Velazquez E, Leijenaar R, et al (2012) Radiomics: Extracting more information from medical images using advanced feature analysis. Eur J Cancer Oxf Engl 1990 48:441-446. https://doi.org/10.1016/j.ejca.2011.11.036

2. Lambin P, Leijenaar RTH, Deist TM, et al (2017) Radiomics: the bridge between medical imaging and personalized medicine. Nat Rev Clin Oncol 14:749-762. https://doi.org/10.1038/nrclinonc.2017.141

3. Villanueva-Meyer JE, Mabray MC, Cha S (2017) Current Clinical Brain Tumor Imaging. Neurosurgery 81:397-415. https://doi.org/10.1093/neuros/nyx103

4. Chaddad A, Kucharczyk MJ, Daniel P, et al (2019) Radiomics in Glioblastoma: Current Status and Challenges Facing Clinical Implementation. Front Oncol 9:374. https://doi.org/10.3389/fonc.2019.00374

5. Singh G, Manjila S, Sakla N, et al (2021) Radiomics and radiogenomics in gliomas: a contemporary update. Br J Cancer 125:641-657. https://doi.org/10.1038/s41416-021-01387-w

6. Stupp R, Mason WP, van den Bent MJ, et al (2005) Radiotherapy plus concomitant and adjuvant temozolomide for glioblastoma. N Engl J Med 352:987-996. https://doi.org/10.1056/NEJMoa043330

7. Tseng C-L, Stewart J, Whitfield G, et al (2020) Glioma consensus contouring recommendations from a MR-Linac International Consortium Research Group and evaluation of a CT-MRI and MRI-only workflow. J Neurooncol 149:305-314. https://doi.org/10.1007/s11060-020-03605-6

8. Perry JR, Laperriere N, O'Callaghan CJ, et al (2017) Short-Course Radiation plus Temozolomide in Elderly Patients with Glioblastoma. N Engl J Med 376:1027-1037. https://doi.org/10.1056/NEJMoa1611977

9. Isensee F, Schell M, Pflueger I, et al (2019) Automated brain extraction of multisequence MRI using artificial neural networks. Hum Brain Mapp 40:4952-4964. https://doi.org/10.1002/hbm.24750

10. Jenkinson $M$, Smith $S$ (2001) A global optimisation method for robust affine registration of brain images. Med Image Anal 5:143-156. https://doi.org/10.1016/S1361-8415(01)00036-6

11. Jenkinson M, Bannister P, Brady M, Smith S (2002) Improved Optimization for the Robust and Accurate Linear Registration and Motion Correction of Brain Images. Neurolmage 17:825-841. https://doi.org/10.1006/nimg.2002.1132

12. Greve DN, Fischl B (2009) Accurate and robust brain image alignment using boundary-based registration. Neurolmage 48:63-72. https://doi.org/10.1016/j.neuroimage.2009.06.060

13. Tustison NJ, Avants BB, Cook PA, et al (2010) N4ITK: improved N3 bias correction. IEEE Trans Med Imaging 29:1310-1320. https://doi.org/10.1109/TMI.2010.2046908

14. Beare R, Lowekamp B, Yaniv Z (2018) Image Segmentation, Registration and Characterization in R with SimplelTK. J Stat Softw 86:. https://doi.org/10.18637/jss.v086.i08 
15. Nyúl LG, Udupa JK (1999) On standardizing the MR image intensity scale. Magn Reson Med 42:1072-1081. https://doi.org/10.1002/(sici)1522-2594(199912)42:6<1072::aid-mrm11>3.0.co;2-m

16. Nyul LG, Udupa JK, Zhang X (2000) New variants of a method of MRI scale standardization. IEEE Trans Med Imaging 19:143-150. https://doi.org/10.1109/42.836373

17. Knight J, Taylor GW, Khademi A (2017) Equivalence of histogram equalization, histogram matching and the Nyul algorithm for intensity standardization in MRI. J Comput Vis Imaging Syst 3:. https://doi.org/10.15353/vsnl.v3i1.170

18. Yushkevich PA, Piven J, Hazlett HC, et al (2006) User-guided 3D active contour segmentation of anatomical structures: significantly improved efficiency and reliability. Neurolmage 31:1116-1128. https://doi.org/10.1016/j.neuroimage.2006.01.015

19. Griethuysen JJM van, Fedorov A, Parmar C, et al (2017) Computational Radiomics System to Decode the Radiographic Phenotype. Cancer Res 77:e104-e107. https://doi.org/10.1158/0008-5472.CAN17-0339

20. Team RC (2021) R: A language and environment for statistical computing. R Foundation for Statistical Computing, Vienna, Austria

21. Max Kuhn (2020) caret: Classification and Regression Training

22. Friedman JH, Hastie T, Tibshirani R (2010) Regularization Paths for Generalized Linear Models via Coordinate Descent. J Stat Softw 33:1-22. https://doi.org/10.18637/jss.v033.i01

23. Simon N, Friedman J, Hastie T, Tibshirani R (2011) Regularization Paths for Cox's Proportional Hazards Model via Coordinate Descent. J Stat Softw 39:1-13. https://doi.org/10.18637/jss.v039.i05

24. Terry M Therneau (2020) A Package for Survival Analysis in R

25. Therneau TM, Grambsch PM (2000) Modeling Survival Data: Extending the Cox Model. Springer, New York, NY

26. Simon RM, Subramanian J, Li M-C, Menezes S (2011) Using cross-validation to evaluate predictive accuracy of survival risk classifiers based on high-dimensional data. Brief Bioinform 12:203-214. https://doi.org/10.1093/bib/bbr001

27. Patrick J. Heagerty, Paramita Saha-Chaudhuri (2012) risksetROC: Riskset ROC curve estimation from censored survival data

28. Kuo MD, Jamshidi N (2014) Behind the numbers: Decoding molecular phenotypes with radiogenomics-guiding principles and technical considerations. Radiology 270:320-325. https://doi.org/10.1148/radiol.13132195

29. Bagher-Ebadian H, Siddiqui F, Liu C, et al (2017) On the impact of smoothing and noise on robustness of CT and CBCT radiomics features for patients with head and neck cancers. Med Phys 44:1755-1770. https://doi.org/10.1002/mp.12188

30. Qin Q, Shi A, Zhang R, et al (2020) Cone-beam CT radiomics features might improve the prediction of lung toxicity after SBRT in stage I NSCLC patients. Thorac Cancer 11:964-972. https://doi.org/10.1111/1759-7714.13349

Page 12/19 
31. Prasanna P, Patel J, Partovi S, et al (2017) Radiomic features from the peritumoral brain parenchyma on treatment-naïve multi-parametric MR imaging predict long versus short-term survival in glioblastoma multiforme: Preliminary findings. Eur Radiol 27:4188-4197. https://doi.org/10.1007/s00330-016-4637-3

32. Dasgupta A, Geraghty B, Maralani PJ, et al (2021) Quantitative mapping of individual voxels in the peritumoral region of IDH-wildtype glioblastoma to distinguish between tumor infiltration and edema. J Neurooncol 153:251-261. https://doi.org/10.1007/s11060-021-03762-2

33. Malik N, Geraghty B, Dasgupta A, et al (2021) MRI radiomics to differentiate between low grade glioma and glioblastoma peritumoral region. J Neurooncol 155:181-191. https://doi.org/10.1007/s11060-021-03866-9

34. Bae S, Choi YS, Ahn SS, et al (2018) Radiomic MRI Phenotyping of Glioblastoma: Improving Survival Prediction. Radiology 289:797-806. https://doi.org/10.1148/radiol.2018180200

35. Azoulay M, Chang SD, Gibbs IC, et al (2020) A phase I/II trial of 5-fraction stereotactic radiosurgery with 5-mm margins with concurrent temozolomide in newly diagnosed glioblastoma: primary outcomes. Neuro-Oncol 22:1182-1189. https://doi.org/10.1093/neuonc/noaa019

\section{Tables}

Table 1 


\begin{tabular}{|c|c|c|c|c|}
\hline Characteristic & All Patients & GTR & STR & Biopsy \\
\hline No. of Patients & $235(100 \%)$ & $57(24 \%)$ & $118(50 \%)$ & $60(26 \%)$ \\
\hline \multicolumn{5}{|l|}{ Gender } \\
\hline Male & $149(63 \%)$ & $35(61 \%)$ & $75(64 \%)$ & $39(65 \%)$ \\
\hline Female & $86(37 \%)$ & $22(39 \%)$ & $43(36 \%)$ & $21(35 \%)$ \\
\hline \multicolumn{5}{|l|}{ Age (years) } \\
\hline Median (Range) & $63(20-84)$ & $60(20-81)$ & $64(39-84)$ & $63(34-83)$ \\
\hline \multicolumn{5}{|l|}{ GTV (cc) } \\
\hline Median (Range) & $31.3(1.19-170)$ & $18.2(1.19-89.0)$ & $33.3(7.18-170)$ & $37.4(3.03-158)$ \\
\hline \multicolumn{5}{|l|}{ MGMT } \\
\hline Methylated & $49(21 \%)$ & $13(23 \%)$ & $25(21 \%)$ & $11(18 \%)$ \\
\hline Unmethylated & $55(23 \%)$ & $9(16 \%)$ & $31(26 \%)$ & $15(25 \%)$ \\
\hline Unknown & $131(56 \%)$ & $35(61 \%)$ & $62(53 \%)$ & $34(57 \%)$ \\
\hline \multicolumn{5}{|c|}{ Steroid use after surgery } \\
\hline$<2$ weeks & $31(13 \%)$ & $9(16 \%)$ & $15(13 \%)$ & $7(12 \%)$ \\
\hline 2 weeks or more & $183(78 \%)$ & $41(72 \%)$ & $91(77 \%)$ & $51(84 \%)$ \\
\hline None & $7(3 \%)$ & $3(5 \%)$ & $3(2 \%)$ & $1(2 \%)$ \\
\hline Unknown & $14(6 \%)$ & $4(7 \%)$ & $9(8 \%)$ & $1(2 \%)$ \\
\hline \multicolumn{5}{|c|}{ Interval surgery and planning MRI (days) } \\
\hline Median (Range) & $19(4-48)$ & $22(9-35)$ & $20(5-46)$ & $15(4-48)$ \\
\hline \multicolumn{5}{|l|}{ Radiation } \\
\hline Conventional & $132(56 \%)$ & $38(67 \%)$ & $68(58 \%)$ & $26(43 \%)$ \\
\hline Hypofractionated & $103(44 \%)$ & $19(33 \%)$ & $50(42 \%)$ & $34(57 \%)$ \\
\hline \multicolumn{5}{|c|}{ Concurrent Temozolomide } \\
\hline Yes & $214(91 \%)$ & $55(97 \%)$ & $108(92 \%)$ & $51(85 \%)$ \\
\hline No & $21(9 \%)$ & $2(3 \%)$ & $10(8 \%)$ & $9(15 \%)$ \\
\hline \multicolumn{5}{|c|}{ Adjuvant Temozolomide } \\
\hline Yes & $152(65 \%)$ & $48(84 \%)$ & $74(63 \%)$ & $30(50 \%)$ \\
\hline No & 83 (35\%) & $9(16 \%)$ & 44 (37\%) & 30 (50\%) \\
\hline
\end{tabular}




\section{Figures}

1) Resampling RT Planning Contours

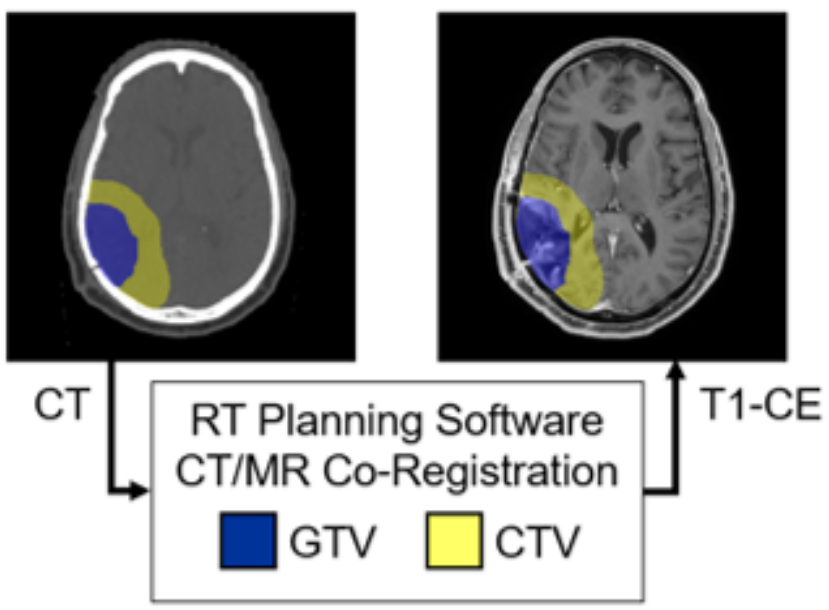

3) Image Preprocessing

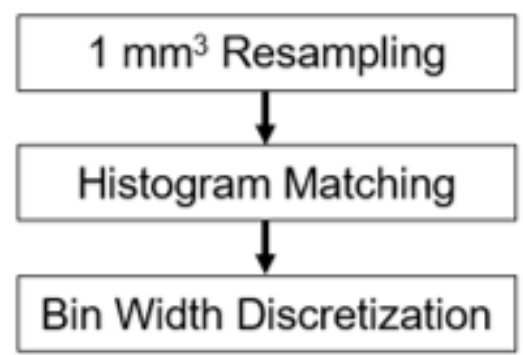

2) Skull Stripping \& Registration
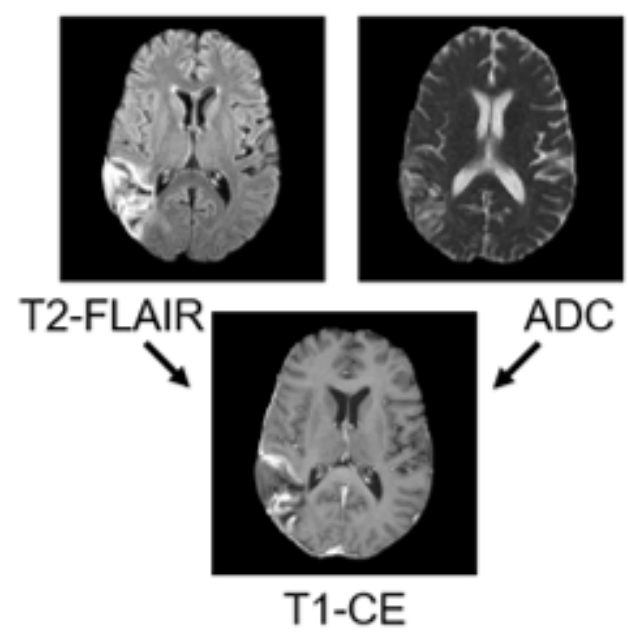

4) Feature Extraction

First-Order (18)

GLCM (22)

GLDM (14)

GLRLM (16)

GLSZM (16)

NGTDM (5)

Figure 1

Workflow for image preprocessing and feature extraction. 
All Patients

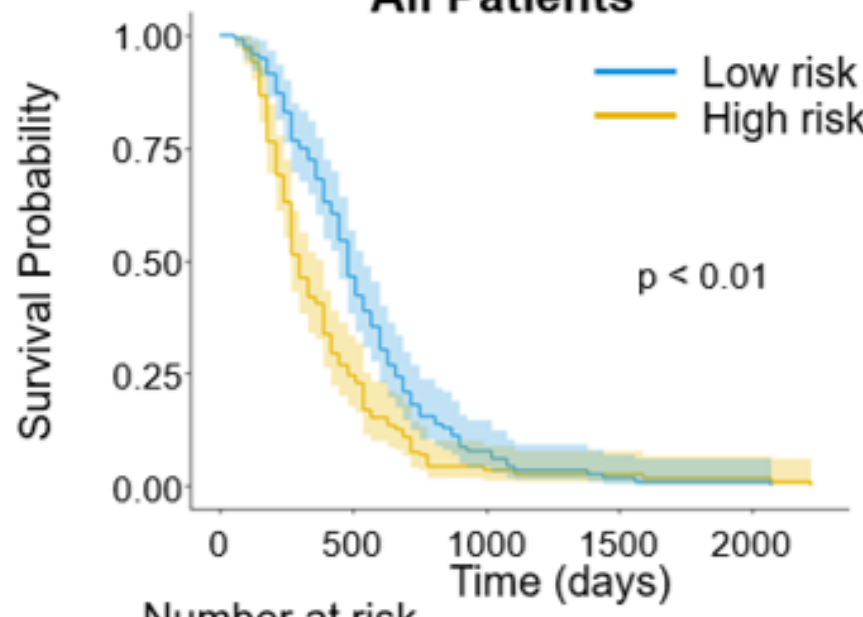

Number at risk

$\begin{array}{cccccc}\text { High risk } & 119 & 29 & 4 & 3 & 2 \\ \text { Low risk } & 116 & 54 & 9 & 2 & 1\end{array}$

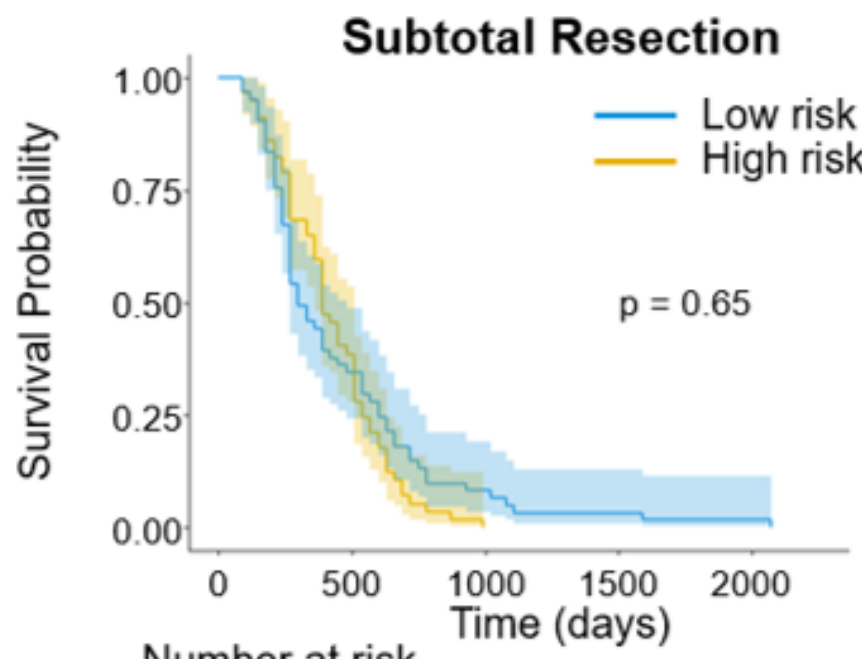

$\begin{array}{llllll}\text { High risk } & 57 & 22 & 0 & 0 & 0\end{array}$

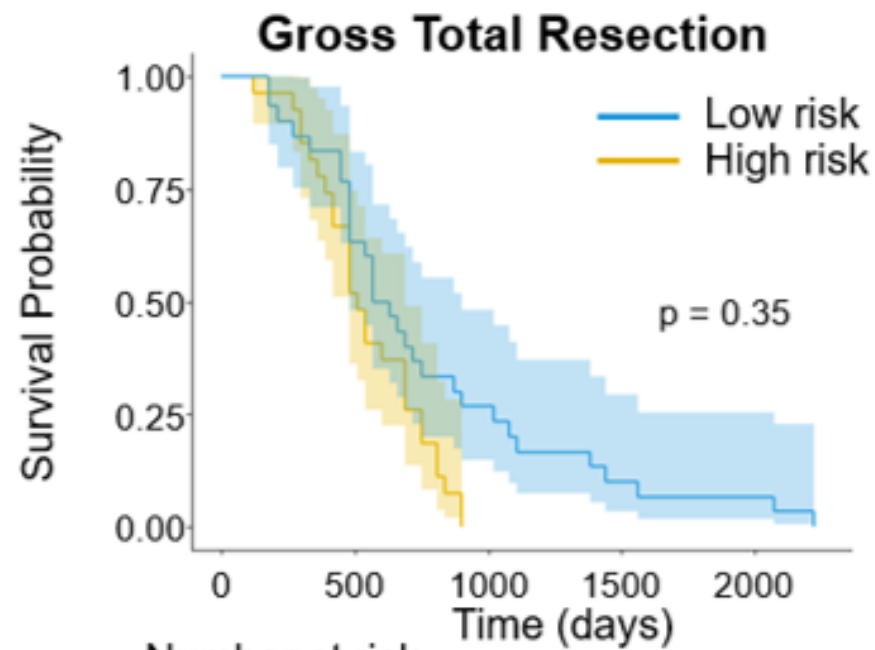

Number at risk

Low risk $30 \quad 19 \quad 3 \quad 3 \quad 3 \quad 2$

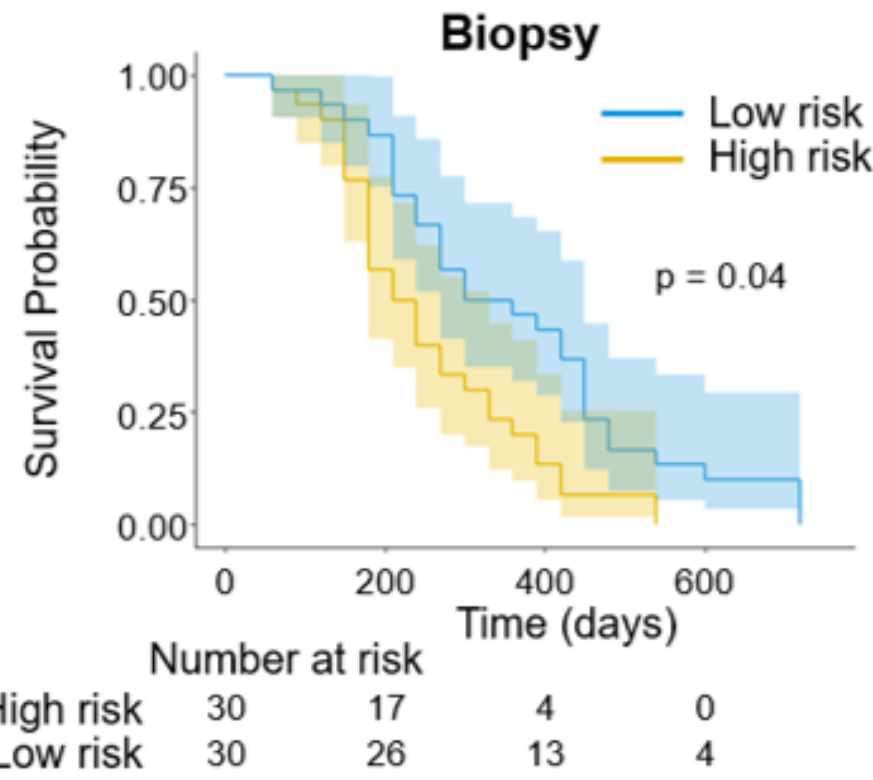

Figure 2

Kaplan-Meier plots for the binary risk-stratified groups based on the radiomics modeling for all the patients and individual surgical subgroups. 


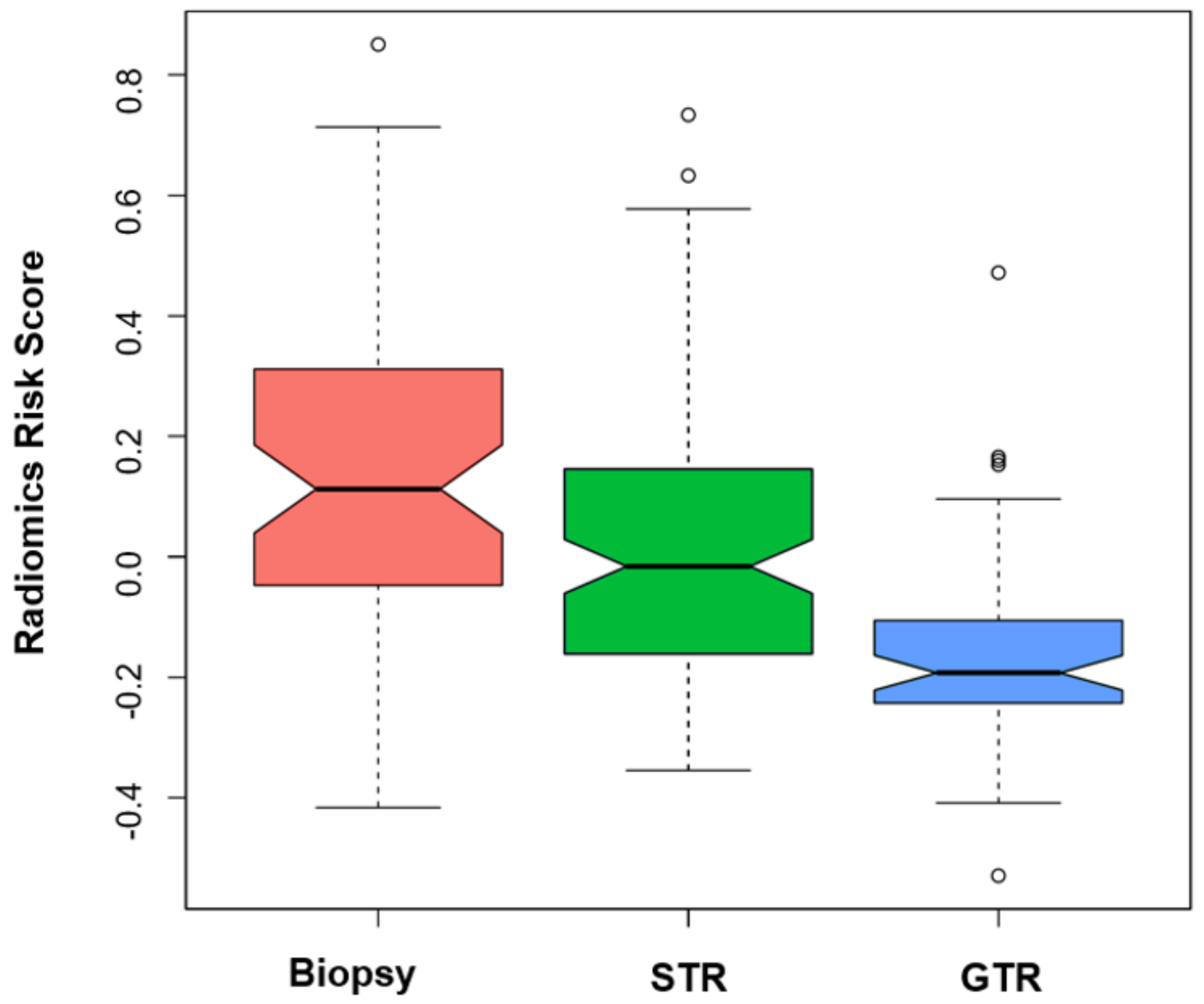

Figure 3

Radiomics risk score across the three surgical subgroups. 

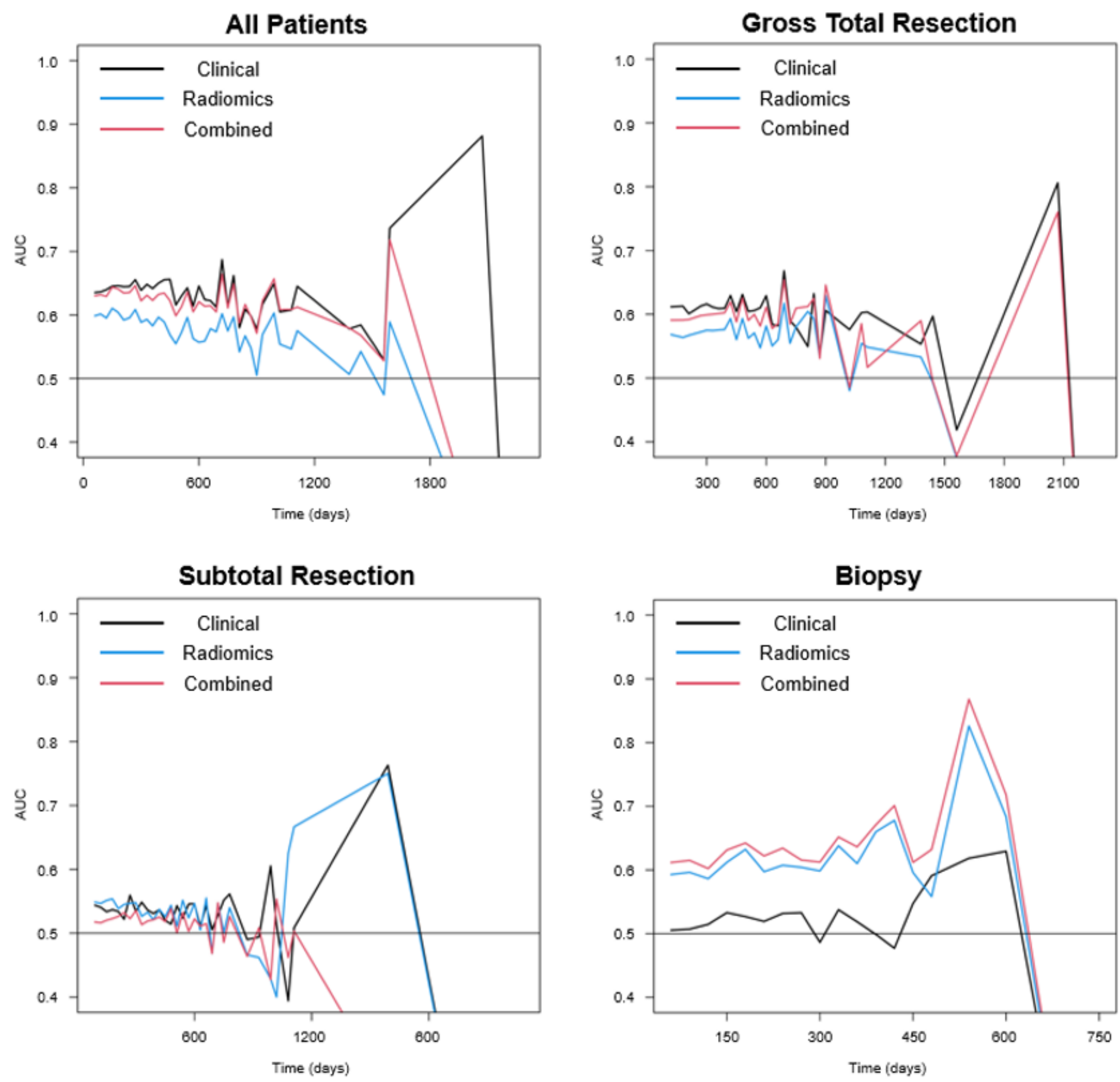

Figure 4

Integrated area under curve values with time as the dependent variable (X-axis) for all the patients and individual surgical subgroups.

\section{Supplementary Files}

This is a list of supplementary files associated with this preprint. Click to download. 
- SupplementaryFigure1.tif

- SupplementaryFigure2.tif 